\title{
DEVELOPMENTAL ANOMALIES
}

NEUROANATONY OF FPAGILE X SYNDROME

Posterior fossa abnormalities measured by magnetic resonance images of the brain were reported in a group of 14 males with fragile $X$ syndrome from the Division of Child Psychiatry, and the Departments of Psychiatry and Radiology, The Johns Hopkins University School of Medicine, and The Kennedy Institute, Baltimore, MD. The size of the posterior cerebellar vermis was significantly decreased and the fourth ventricle significantly increased in males with fragile $X$ syndrome compared to a group of 17 males with other causes of developmental disability and 18 males with normal IQs. The lack of a significant correlation between age and size of vermis argues against atrophy as the cause but rather a developmental hypoplasia of the posterior vermis. (Reiss AL et al. Neuroanatamy of fragile X syndrome: the posterior fossa. Ann Neurol Jan 1991; 29:26-32).

COMENT. Hypoplasia of the cerebellar vermal lobules VI and VII has also been described in patients with autism (Courchesne E et al. N Engl J Med 1988; 318:1349). Aut istic-like behavior occurs frequently in males with fragile $X$ syndrome and the posterior vermis hypoplasia may have a causative relation. The cerebellar vermis plays an important role in the modulation and execution of motor behavior, and hyperactivity and repetitive stereotypic movements are common behaviorisms in fragile $X$ patients. Language dysfunction also occurs with fragile $X$ syndrome and the posterior vermis of the cerebellum is involved in auditory processing of language. So-called "cerebellar mutism" may develop after removal of posterior fossa tumors particularly medulloblastama, and this complication has been correlated with the amount of the posterior vermis resected. (Platenberg C et al. AJNR 1989; 10:891). A seven year old girl with a midline posterior fossa tumor developed mutism postoperatively after removal of an astrocytoma that involved the vermis of the cerebellum. Recovery was slow but speech was regained after a two month period of therapy (Millichap JG, McLone D, unpublished case report).

TUBEROUS SCLEROSIS AND NEONATAL ASTROCYTOMA

The relative value of neural imaging studies in the diagnosis of a neonatal astrocytoma associated with tuberous sclerosis is reported from the Departments of Neurosciences, Pediatrics, and Pathology, University of California, San Diego, CA. The infant was hypotonic at birth and had poor respiratory effort. Apgar scores were 3/8. Motor examination revealed decreased tone in proximal upper extremities and neck. Deep tendon reflexes were generally exaggerated and plantar responses were extensor bilaterally. Wood's lamp examination was equivocal. There was no family history of tuberous sclerosis or epilepsy and the parents showed no neurocutaneous stigmata. An echoencephalogram revealed a large mass in the left lateral ventricle arising from the left caudate nucleus and obstructing the foramen of Monroe. 
The study was consistent with an intraventricular tumor rather than a hematoma. The MRI revealed a large irregular lobulated mass believed to be a tumor arising from the corpus callosum, possibly a lipoma. The CT revealed a nonenhancing, hyperdense mass in the left frontal horn interpreted as an intraventricular hemorrhage. A CT-guided needle biopsy of the mass showed gemistocytic cells resembling large reactive astrocytes. A cardiac echocardiogram revealed multiple nodules in the intraventricular septum, consistent with multiple rhabdonyomas. Funduscopic exam revealed two small retinal hamartomas in the right eye. Ultrasound of the kidneys was negative. At eight weeks of age the infant had developed multiple hypopigmented macules on trunk and extremities. (Hahn JS et al. Neonatal subependymal giant cell astrocytoma associated with tuberous sclerosis: MRI, CT, and ultrasound correlation. Neurology Jan 1991; 41:124-128).

COMMENT. The interpretation of the echoencephalogram was closer to the correct diagnosis than that of the MRI and CT. Even without the needle biopsy of the mass the diagnosis of tuberous sclerosis and associated tumor may have been suspected and almost certainly determined by the echocardiogram and cardiac rhabdomyomas. Echoencephalogram may be the diagnostic study of choice in newborns. The MRI picture is atypical showing decreased signal intensity on T2-weighted imaging and increased signal intensity on T1-weighted studies.

\section{SPASTIC DIPLEGIA AND THE MRI}

The MRI findings in 34 children with spastic diplegia examined between 2 and 10 years of age are reported from the Seirei-Hamamatsu General Hospital, Mikatabara, Hammatsu, Shi zuoka; Nagoya City Medical School; and the National Rehabilitation Center for Disabled Children, Tokyo, Japan. There was dilation of the trigone, atrophy of the peritrigonal white matter, and prominent deep cortical sulci. The appearance of cerebral white matter was classified into four patterns: 1) normal; 2) peritrigonal white matter showing a thin contiguous line in one hemisphere; 3 ) peritrigonal white matter reduced in both hemispheres; and 4) the reduction of the white matter expanded anteriorly from the trigones to the bodies of the lateral ventricles. The degree of cerebral white matter loss correlated with severity of motor disability but not with severity of mental impairment. The distribution of periventricular high intensity T2-weighted images did not correlate with motor or mental disability. (Yokochi $\mathrm{K}$ et al. Magnetic resonance imaging in children with spastic diplegia: Correlation with the severity of their motor and mental abnormality. Dev Med Child Neurol Jan $1991 ; 33: 18-25)$.

COMMENT. The MRI findings were similar to those reported with periventricular leukomalacia. In infancy leukomalacia is characterized by delayed myelination and demonstrated as high intensity areas on T2 weighted images. It occurs most commonly in preterm infants and all but three of the children in this study had birth weights lower than 2000 grams. Mild degrees of periventricular white matter reduction are of ten interpreted as a normal variant in children, but correlations with delay in motor development should be considered. 